\title{
Simulation study of intra- and intermicellar ordering in triblock-copolymer systems
}

\author{
C. M. Wijmans and E. Eiser \\ van 't Hoff Institute for Molecular Science, University of Amsterdam, Nieuwe Achtergracht 166, \\ 1018 WV Amsterdam, The Netherlands \\ D. Frenkel \\ FOM Institute for Atomic and Molecular Physics, Kruislaan 407, 1098 SJ Amsterdam, The Netherlands
}

(Received 11 November 2003; accepted 29 December 2003)

\begin{abstract}
We report a numerical study of the structure and phase behavior of a model for a triblock-copolymer solution. The aim of this study is to investigate the nature of the dense micellar phase that can form in such systems. The simulations were performed on a lattice model for PEO (poly(ethylene-oxide))-PPO (poly(propylene-oxide))-PEO polymers. At high volume fractions, the structure factor of the amphiphile-solvent system can be mapped onto that of a monodisperse hard-sphere fluid. Yet, a low-density hard-sphere model cannot account for the properties of the dilute micellar solution. Moreover, direct inspection of the snapshots of the suspension show that these model triblock-copolymer micelles are neither hard, nor spherical, nor monodisperse. (C) 2004 American Institute of Physics. [DOI: 10.1063/1.1649730]
\end{abstract}

\section{INTRODUCTION}

When a block copolymer is mixed with a liquid that is a good solvent for one of its constituent monomers, but not for the other, the polymer can assemble into mesoscopic structures, such as micelles, lamellae, or hexagonal phases. Because of this ability to self-assemble into nanoscale structures, block copolymers are used as an ingredient in a variety of industrial and pharmaceutical compounds. A particularly interesting class of block copolymers are the PEO-PPOPEO triblock copolymers (better known under the commercial name of "Pluronics"). These molecules owe their amphiphilic character to the fact that the central poly(propylene-oxide) [PPO] block is more hydrophobic than the terminal poly(ethylene-oxide) [PEO] blocks. And, unlike short surfactant molecules, the hydrophobicity of the middle block increases continuously as the temperature is increased. As a consequence, the phase diagram of the aqueous solution of Pluronics shows a stronger dependence on temperature than on concentration. This is one of the reasons why Pluronics solutions have been studied extensively with a variety of experimental techniques. ${ }^{1-3}$

Mortensen ${ }^{6}$ has reported systematic small-angle neutronscattering experiments of a number of pluronics, including an aqueous solution of $\mathrm{EO}_{25} \mathrm{PO}_{40} \mathrm{EO}_{25}$ (commercial acronym "P85"). With increasing concentration and temperature, this system forms spherical micelles. The experiments clearly show that these micelles are dynamical objects (they can exchange polymer chains with the solvent), yet the structural properties appear to be those of a hard-sphere fluid. In fact, the micellar solution even appears to undergo a hardsphere freezing transition at a micellar volume fraction of $47 \%$. The volume fraction of the coexisting micellar crystal is $53 \%$. These numbers are quite close to those of pure hard spheres (respectively, $49.4 \%$ and $54.5 \%$ ). This finding is surprising because, on a microscopic scale, the micelles do not resemble hard spheres. The experiments suggest that, while the micellar liquid is structurally identical to a hard-sphere liquid, the crystalline phase that forms is qualitatively different from a hard-sphere crystal. In fact, the scattering experiments suggest that the "crystal" phase has long-ranged bond order, but no long-ranged translational order. The ordered phase should therefore be called cubatic, rather than cubic. $^{4,5,7}$ The behavior observed for P85 is, presumably, representative for a larger number of PEO-PPO-PEO triblock copolymers (be it that the structure of the ordered phase can be either fcc-like or bcc-like, depending on the length of the PEO tails).

The aim of the present paper is to gain a better understanding of the nature of dense micellar solutions of triblock copolymers. In particular, we wish to investigate to what extent the micelles are hard-sphere-like in their individual and collective behavior.

As a fully atomistic simulation of the phase behavior of a system consisting of hundreds of micelles in aqueous solution is (at present) prohibitively expensive, one of our aims is to develop a simple, coarse-grained lattice model that can reproduce the structural properties of a micellar liquid of triblock copolymers.

\section{COARSE-GRAINED MODELS}

Several authors have used self-consistent-field (SCF) theory to model pluronics-water mixtures. ${ }^{8-12}$ In this approach it is assumed that the polymer self-assembles into an aggregate with a fixed geometry (e.g., spherical or cylindrical). The free energy of the aggregate and its structural properties can then be calculated within the mean-field approximation. Predictions of the phase behavior can be made using the SCF model. A disadvantage of this method is that the calculations are based on an equilibrium between an isolated aggregate and a homogeneous polymer-solvent mixture. 
Fluctuations of the individual micelles are not considered and interactions between aggregates are accounted for in a rather crude manner. The method is therefore less suited to study the collective behavior of systems containing many fluctuating micelles.

In order to proceed beyond the mean-field approximation, one can make use of several direct simulation techniques. $^{13,14}$ A variety of coarse-grained models have been developed to simulate surfactant and block copolymer systems on length scales that are far larger than can be simulated using atomistic models. Among these coarse-grained models, lattice models have the advantage that they are sufficiently cheap to allow the modeling of systems, containing many hundreds, or even thousands, of chains. For this reason, there is an extensive literature on simulations of lattice models for copolymer systems. The majority of these papers deal with $A B$ diblock chains, where the $A$ block is hydrophilic (or, more generally, "solvophilic") while interactions between the solvent and the $B$ block are less favorable. ${ }^{15-32}$ Triblock copolymer-solvent systems (either $A B A$ or $B A B$ ), have been the subject of a smaller number of simulation studies. ${ }^{33-36}$

In the context of the present study, the simulations by Kim and Jo of an $A B A$ system ${ }^{35}$ are of direct relevance. Using grand canonical Monte Carlo simulations in combination with a multiple histogram method these authors investigated the micellization process of $A B A$ and $B A B$ block copolymers. In particular they showed that the block length of the less soluble $B$ block determines the critical micelle concentration of the system, and the shape and aggregation number of the micelles. Our study is largely complementary to that of Kim and Jo, as we are primarily interested in the high-density behavior well above the critical micelle concentration (CMC).

The remainder of this paper is organized as follows: in the next section, we describe in some detail the choice of the model parameters and simulation method that we used. Next, we present the simulation results for a model $A B A$ chain, first of all concentrating on the low concentration regime, where the system consists of noninteracting micellar aggregates. Subsequently, we consider the behavior at higher concentrations. As in the experiments, we attempt to analyze the structural properties of these dense micellar systems in terms of the hard-sphere structure factor.

\section{SIMULATION METHODS}

\section{A. Model}

In our simulations, we considered a coarse-grained model for $A B A$ triblock copolymers. The polymer consisted of a central block of $N_{B} B$ segments and two end $A$ blocks of $N_{A}$ segments. In the present simulations, we chose $N_{A}=N_{B}$ $=6$. Clearly, this number of segments is much less than the number of monomers in a real PEO-PPO-PEO chain, in other words: a segment represents a Kuhn length, rather than a monomer. We chose this model (and the interaction parameters described below) because it is one of the simplest systems that gives rise to the formation of multichain micelles at concentrations similar to those used in experiments. More- over, Milchev and Binder ${ }^{37}$ have shown that diblock copolymer systems can be modeled by lattice polymers that contain as few as four segments.

In the model, the chain segments only interact with their nearest-neighbor lattice sites (lattice coordination number $z$ $=6$ ). The total energy of the system is defined by the three contact energies $\epsilon_{A S}, \epsilon_{B S}$, and $\epsilon_{A B}$. As default values for these interaction parameters we use the following set of values: $\epsilon_{A S}=0, \epsilon_{B S}=\epsilon_{A B}=\epsilon=0.8$. All interaction energies are expressed in units of the thermal energy $k_{B} T$. Hence the interaction energies given actually correspond to $\epsilon / k_{B} T$. These values were chosen after conducting some test runs with different interaction values. The value $\epsilon=0.8$ leads to a critical micelle volume fraction of the order of $10^{-2}$, which is comparable to values for nonionic triblock copolymer systems. ${ }^{38}$ In the results section, we discuss in some detail the effect of different interaction parameters on the properties of the system.

The simulations were performed on a cubic lattice consisting of $L^{3}$ lattice sites. Periodic boundary conditions were applied in all three directions. No two segments could occupy the same lattice site. Empty lattice sites represented the solvent $S$ (i.e., the solvent was assumed to be incompressible). The total number of (polymer) segments $N_{s}$ is given by $N_{s}=N_{P} \times\left(2 N_{A}+N_{B}\right)$ and the overall polymer volume fraction $\phi$ is $\phi=N_{s} / L^{3}$.

\section{B. Monte Carlo moves}

The simulations were initiated by placing the chains on the lattice in random conformations. The system was then allowed to equilibrate, using standard Monte Carlo moves for lattice chains. ${ }^{30,32,39}$ We used five different kinds of moves to generate new configurations with the following typical weights: end segment kink flip (7\%), middle segment kink flip (23\%), reptation (40\%), crankshaft move (25\%), Brownian move $(5 \%)$. These frequencies were changed slightly as a function of volume fraction to ensure optimal efficiency. At high volume fraction especially the Brownian motion move becomes very inefficient. The acceptance or rejection of a new configuration was determined using the standard Metropolis acceptance criterion. ${ }^{40}$

In order to keep track of the relaxation "dynamics" of the system we introduce, somewhat arbitrarily, a Monte Carlo "time scale." We chose our Monte Carlo time unit to correspond to ten trial moves per polymer segment.

\section{Structural analysis}

In order to identify aggregates, such as micelles, we assume that two chains belong to the same aggregate if at least one (hydrophobic) $B$ segment on one chain is adjacent to a $B$ segment on the other chain. Using this criterion, we can determine the (normalized) aggregate-size distribution $P\left(N_{\text {agg }}\right)$, where $N_{\text {agg }}$ is the aggregation number.

In addition, we locate the center of mass of each aggregate, and we compute the principal moments of inertia $I_{1}$, $I_{2}$, and $I_{3}$ with respect to this center of mass. In what follows, we order the components of the inertia tensor such that 
$I_{1} \leqslant I_{2} \leqslant I_{3}$. Following Ref. 32, we introduce a single parameter $\alpha_{s}$ (the asphericity parameter) to quantify the shape of an aggregate:

$$
\alpha_{s}=\frac{\left(I_{1}-I_{2}\right)^{2}+\left(I_{1}-I_{3}\right)^{2}+\left(I_{2}-I_{3}\right)^{2}}{\frac{1}{2}\left(I_{1}+I_{2}+I_{3}\right)^{2}} .
$$

In order to gain insight in the average structure of the aggregate, we can compute the radial density distribution of segments within an aggregate.

When simulating equilibrium properties of a system, the computed properties should be averaged over many fluctuation "times." We therefore need to estimate a characteristic relaxation time of the system. In order to do this we introduce two probabilities: $P^{c}(\tau, t)$ and $P^{u}(\tau)$ (the superscripts $c$ and $u$ stand for conditional and unconditional). $P^{u}(\tau)$ is defined as the probability that, at time $\tau$, two randomly chosen chains belong to the same aggregate. $P^{c}(\tau, t)$ is defined as the probability that two chains that were in the same aggregate at time $\tau-t$ still belong to the same aggregate at time $\tau$. This allows us to define a relaxation function $f(t)$ as

$$
f(t)=\left\langle\frac{P^{c}(\tau, t)-P^{u}(\tau)}{1-P^{u}(\tau)}\right\rangle_{\tau} .
$$

Clearly, $f(t)=1$ for $t=0$, and $f(t)=0$ for $t \rightarrow \infty$. The decay time of the function $f(t)$ is a measure of the time scale on which exchange takes place of chains between aggregates.

\section{Radial distribution functions}

At high volume fractions the analysis based on individual aggregates is less informative. Experimentally, the structure of the dense micellar systems is usually studied using scattering techniques. The measured scattering intensities are directly related to the Fourier transform of the radial distribution function of the scattering density. This distribution function measures both intra- and intermolecular correlations. To disentangle both contributions, it is usually necessary to make an assumption about the shape (more precisely, the form factor) of the scatterers. For instance, it is often assumed that the form factor of micelles that is measured at low densities is unchanged as the density is increased. In contrast, in simulations no such assumptions are needed. Thus simulations allow us to test some of the assumptions that are used to extract information about the micellar structure factor from experimental scattering data.

\section{E. Chemical potential}

All simulations presented here were performed at constant $N_{s}, V$, and $T$. To determine the chemical potential of the amphiphile chains we used a test chain insertion method (the Widom method). ${ }^{41,42}$ Random conformations of a (nonself-avoiding) chain are generated and inserted into the system. However, at higher volume fractions, this method fails because of the low probability of accepting a trial insertion. As an alternative, we use a test-particle insertion method based on the Rosenbluth algorithm ${ }^{45}$ to generate trial conformations. ${ }^{42-44}$ In this algorithm, the Rosenbluth factor

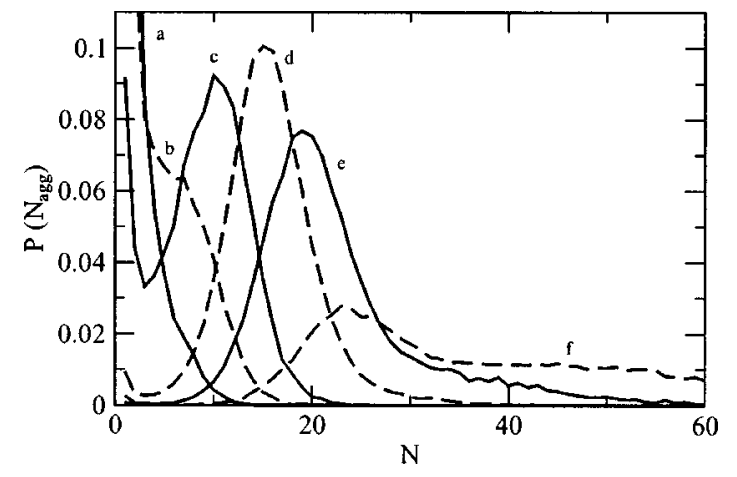

FIG. 1. Aggregate size distribution curves for an $A_{6} B_{6} A_{6}$ triblock chain with interaction parameters $\epsilon_{A S}=0, \epsilon_{B S}=\epsilon_{A B}=0.8$. The amphiphile volume fraction $\phi$ for the various curves is: $\phi=0.009$ (a); 0.021 (b); 0.072 (c); 0.288 (d); 0.432 (e); 0.576 (f).

$W$ is computed for a large number of trial conformations. The excess chemical potential $\mu_{\mathrm{ex}}$ is related to the average value of $W:^{42}$

$$
\mu_{\mathrm{ex}}=-\ln \langle W\rangle \text {. }
$$

The total chemical of the amphiphile chains is the sum of this excess term and the ideal gas term $\mu_{\mathrm{id}}=\ln N_{P} / V$, where $N_{P}$ is the number of polymers in volume $V$.

\section{RESULTS AND DISCUSSION}

\section{A. Aggregate size and shape}

Using the interaction parameters discussed above $\left(\epsilon_{A S}\right.$ $=0, \epsilon_{B S}=\epsilon_{A B}=\epsilon=0.8$ ), we performed a series of simulations. Figure 1 shows how the size distribution of the aggregates changes with the polymer concentration. The different curves represent different volume fractions $\phi$ ranging from 0.009 to 0.576 . The number of chains $N_{P}$ in the simulations ranged from 500 to 4000 . The smallest system size was $L$ $=50$ and the largest $L=100$.

In our analysis, we implicitly assume that the system does not undergo a liquid-liquid demixing transition with increasing density. This assumption is not completely obvious. In fact, Panagiotopoulos et al. ${ }^{36}$ have studied the phase behavior of a number lattice models for di- and triblock surfactant systems. They observed that these systems would either phase separate or undergo a micellization transition, but never both. The model that we use differs from the ones used in Ref. 36 (different coordination number) but, as we argued above, this does not lead to qualitative differences in the phase behavior. If this assumption is correct, then the results of Ref. 36 suggest that the present system should undergo micellization only. Indeed, even in the largest systems that we studied, we never saw evidence for spontaneous demixing. Moreover, the density dependence of the chemical potential (see Fig. 9 below) also suggests that the system does not undergo a first-order phase transition.

At the lowest volume fraction considered in Fig. 1 the aggregate size distribution $P\left(N_{\text {agg }}\right)$ is a monotonically decreasing function of $N_{\text {agg }}$. There exist several ways to define the critical micelle concentration $(\mathrm{CMC}){ }^{31}$ We will use the definition of Ruckenstein and Nagarajan ${ }^{46}$ who identify the 


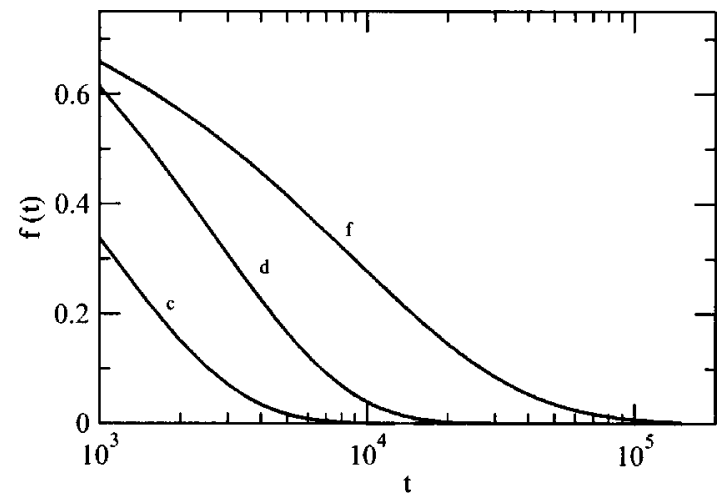

FIG. 2. Relaxation function $f(t)$ [see Eq. (2)] for three of the curves in Fig. 1 (c: $\phi=0.072 ; \mathrm{d}: \phi=0.288 ; \mathrm{f}: \phi=0.576$ ).

CMC with the concentration where the aggregate size distribution exhibits a point of inflection. We find that the CMC occurs around $\phi \approx 0.02$. Above this CMC, the size distribution is approximately symmetric around the most probable aggregation number. As the volume fraction increases, the average aggregate size increases. This behavior is different from that observed in most systems containing micelles of small surfactants. There the number of micelles increases above the $\mathrm{CMC}$, but the (average) aggregation number remains nearly constant. The strong dependence of the aggregate size on the surfactant volume fraction that we observe is surprising. It is tempting to speculate that other triblock copolymers may exhibit similar behavior. However, we did not investigate this.

Figure 1 shows that, at higher concentrations, the distribution function develops a tail. Above $\phi \approx 0.5$ an appreciable fraction of the amphiphiles are incorporated in far larger structures than the aggregates that are formed at low concentrations. The assumption that one can describe the system as an assembly of polydisperse (small) micelles in equilibrium with a low concentration of unimers, dimers, etc., breaks down at these high volume fractions. For $\phi=0.576$ (the largest volume fraction shown in Fig. 1) the aggregate size distribution decays slowly to zero, with a few aggregates containing more than 200 chains. Despite the presence of such large aggregates, the aggregate-size distribution curve at $\phi$ $=0.576$ still shows a (small) maximum at an aggregation number of 23. At higher volume fractions $(\phi=0.7)$ one finds a continuous distribution of sizes up to the maximum size $N_{\text {agg }}=N_{P}$, where all chains in the system belong to a single aggregate (not shown in the graph). Clearly, in this limit, the system cannot be described as a collection of discrete aggregates.

Figure 2 shows the relaxation function $f(t)$ defined by Eq. (2) for several of the volume fractions plotted in Fig. 1. In principle, the Monte Carlo technique that we use to solve the simulation model, provides only equilibrium information. We have therefore not attempted to extract information about the kinetics of our system. It is, however, necessary to check whether the simulation runs are sufficiently long to provide a representative sampling of the available configuration space. As the amphiphile volume fraction increases beyond the $\mathrm{CMC}$, longer simulation times are needed for individual

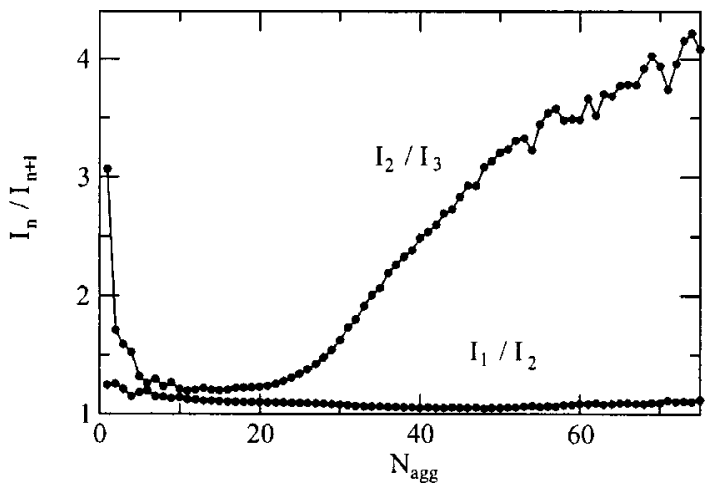

FIG. 3. The average ratios of the principal moments of inertia, $\left\langle I_{1} / I_{2}\right\rangle$ and $\left\langle I_{2} / I_{3}\right\rangle$, of the aggregates of curve $\mathrm{f}(\phi=0.576)$ in Fig. 1.

chains to move from one aggregate to another. Simulation runs of $3 \times 10^{5}$ "time" steps were used to compute the data presented in Fig. 1. This is significantly longer than the corresponding relaxation times of these systems.

Figure 1 provides information on the size (aggregation number) of the aggregates only. Any two chains whose $B$ blocks are direct neighbors of each other are assumed to belong to the same aggregate. No further assumptions are made about the shape of these aggregates. Information on the (average) shape of the aggregates is given in Fig. 3. This figure was obtained by analyzing the aggregates formed at a volume fraction $\phi=0.576$. At this high amphiphile concentration aggregates exist over a wide size range. Their shapes may, however, differ from the shapes of aggregates of similar size at lower volume fractions. This will be studied below. The rather large fluctuations that are seen at high aggregation numbers are due to the fact that one is averaging over a relatively small number of aggregates. In Fig. 3 the ratios $\left\langle I_{1} / I_{2}\right\rangle$ and $\left\langle I_{2} / I_{3}\right\rangle$ of the principal moments of inertia have been averaged for each aggregation number. We could also have plotted the ratios of the average moments of inertia, $\left\langle I_{1}\right\rangle /\left\langle I_{2}\right\rangle$ and $\left\langle I_{2}\right\rangle /\left\langle I_{3}\right\rangle$, but this does not result in a qualitatively different picture. At low aggregation numbers the ratios of the moments of inertia are only slightly larger than unity. This indicates that the micelles are fairly spherical micelles. At higher aggregation numbers $I_{2} / I_{1}$ increases strongly. A similar trend has been observed in amphiphilic diblock chains. ${ }^{25}$

Figure 4 shows the shape of the aggregates (represented by the asphericity parameter $\alpha_{s}$ [Eq. (1)] for a range of volume fractions. We use the rule of thumb that aggregates are effectively spherical if $\alpha_{s}<0.1$. This is the case for aggregates with aggregation numbers up to approximately 20 or 30. For $\phi=0.072$ (curve c) the most prevalent aggregates [i.e., those corresponding to the peak in the distribution curve $\left.P\left(N_{\text {agg }}\right)\right]$, are effectively spherical $\left(\alpha_{s} \approx 0.05\right)$. However, in the tail of the distribution ( $N_{\text {agg }}$ up to 20) the value of $\alpha_{s}$ doubles. As the amphiphile volume fraction increases, the peak in the distribution curve shifts to higher aggregation numbers. Interestingly, the aggregates remain spherical up to larger aggregation numbers. In other words: the shape of aggregates of a fixed size depends on concentration. In the analysis of experiments on real triblock copolymers, it is 


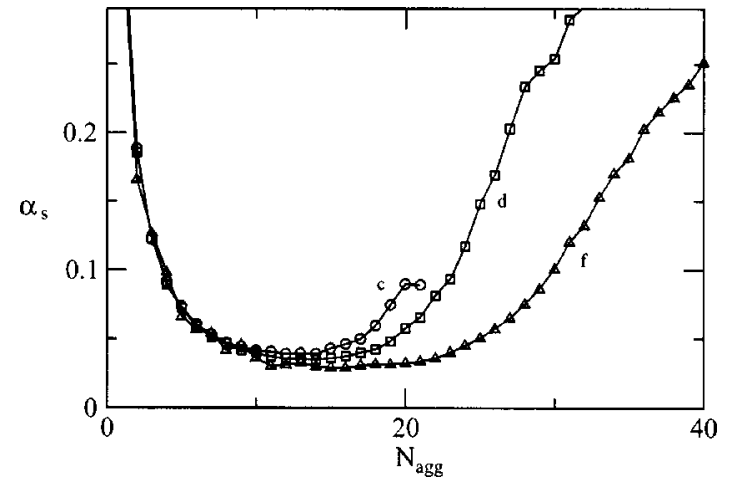

FIG. 4. The asphericity parameter $\alpha_{s}$ [see Eq. (1)] as a function of aggregation number for curves $\mathrm{c}(\phi=0.072), \mathrm{d}(\phi=0.288)$, and $\mathrm{f}(\phi=0.576)$ in Fig. 1.

common practice to obtain the structure factor by dividing the (high-concentration) scattering intensity by the form factor obtained at low concentrations. If the behavior of our model system is representative of real triblock copolymer solutions, this procedure is clearly questionable because we find that both the average size (Fig. 1) and the average shape (Fig. 4) of the micelles do depend on concentration.

For $\phi=0.576$ very large aggregates are formed in addition to the small ones that exist at lower volume fraction. These large aggregates are highly nonspherical.

Figure 5 shows the (average) radial density profiles of two aggregates. In Fig. 5(A) the aggregation number is $N_{\text {agg }}=10$ and the aggregates were simulated at an overall volume fraction $\phi=0.072$. In Fig. 5(B) $N_{\text {agg }}=24$ and $\phi$ $=0.576$. In both cases the asphericity parameter is small, so that it makes sense to plot the radial density with respect to the center of mass (of the $B$ core) of the aggregates. As expected for a micellar aggregate, we observe a radial density profiles that shows a rather diffuse "corona" of $A$ segments around a compact core of $B$ segments.

\section{B. Changing the interaction parameters}

The parameter values that were used for the simulations were chosen after a series of trial simulations in which the effect of the block lengths and interaction parameters were explored. We settled on these parameter values because they lead to a $\mathrm{CMC}$ at a volume fraction that is in the range of

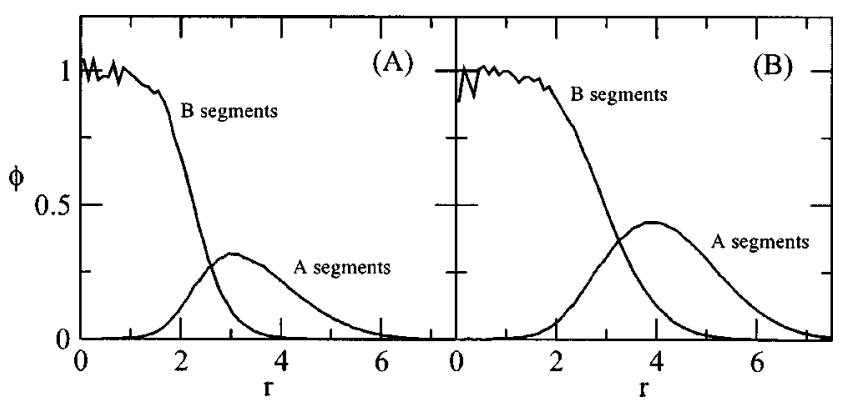

FIG. 5. Density profiles of two micellar aggregates. The length scale $r$ denotes the distance to the center of mass of the core of $B$ segments. Aggregation number $N_{\text {agg }}=10(\mathrm{~A})$ and $N_{\mathrm{agg}}=24$ (B); volume fraction $\phi$ $=0.072(\mathrm{~A})$ and $\phi=0.576(\mathrm{~B})$.

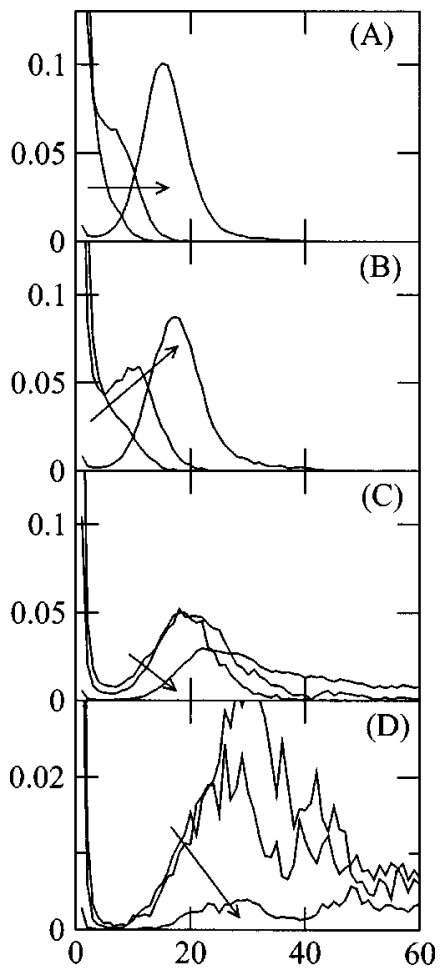

FIG. 6. Aggregate size distribution curves for an $A_{6} B_{6} A_{6}$ triblock chain with interaction parameters $\epsilon_{B S}=\epsilon_{A B}=0.8$. The value of $\epsilon_{A S}$ increases from (A) to (D): $\epsilon_{A S}=0$ (A), $\epsilon_{A S}=0.05$ (B), $\epsilon_{A S}=0.15$ (C), and $\epsilon_{A S}=0.2$ (D). Curves have been plotted for three volume fractions $\phi=0.009,0.021$, and 0.288 . The arrows indicate the direction in which $\phi$ increases.

what one finds experimentally for nonionic triblock copolymers. Furthermore, above the CMC aggregates are formed with a fairly narrow size distribution.

The $A$ segments have a zero interaction energy with the solvent, which is the behavior expected if the hydrophilic segments are chemically similar to the solvent. However, when the hydrophilic block consists of polyethylene-oxide, its interaction with water is temperature dependent: PEO is less hydrophilic at higher temperatures. Figure 6 shows the effect of a repulsive interaction $\left(\epsilon_{A S}>0\right)$ between the $A$ segments and the solvent on the aggregation behavior in the lattice model. Figure 6(A) shows, for three different volume fractions, the aggregate-size distribution curves of Fig. 1 $\left(\epsilon_{A S}=0\right)$. When $\epsilon_{A S}=0.05$ [Fig. 6(B)] the amphiphilic chains clearly have a stronger tendency to aggregate. This is demonstrated by the peak in the curve for $\phi=0.021$. As the repulsion increases further, we see that for $\epsilon_{A S}=0.15$ the amphiphile aggregates even at the lowest volume fraction considered. This tendency of the CMC to shift to lower density as the PEO becomes more hydrophobic, is also observed in experiment. ${ }^{6}$ In addition, the cluster-size distribution starts to develop a prominent tail at large aggregation numbers. At the highest repulsion $\left[\epsilon_{A S}=0.2\right.$, Fig. $\left.6(\mathrm{D})\right]$ the chains are mainly found in very large clusters rather than in small micellar aggregates. This is presumably the finite-system equivalent of the phase separation observed in experiments at high temperatures.

In what follows, we focus our attention on the system with a zero interaction between the end-block segments and 


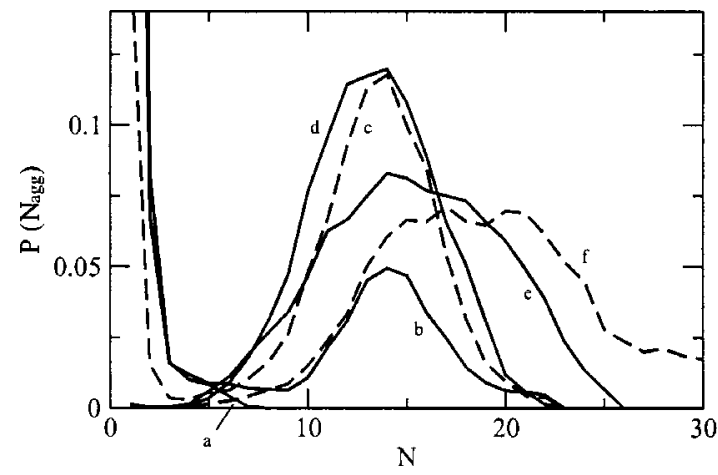

FIG. 7. Aggregate size distribution curves for an $A_{6} B_{6} A_{6}$ triblock chain with interaction parameters $\epsilon_{A S}=0, \epsilon_{B S}=\epsilon_{A B}=1.2$. The amphiphile volume fraction $\phi$ for the various curves is $\phi=4.2 \times 10^{-5}$ (a); $8.4 \times 10^{-5}$ (b); 2.1 $\times 10^{-4}$ (c); 0.021 (d); 0.072 (e); 0.288 (f).

the solvent $\left(\epsilon_{A S}=0\right)$. In Fig. 7 we have used a value $\epsilon$ $=1.2$ to characterize the repulsive hydrophobic interaction. All other parameters are the same as in Fig. 1. This larger value for $\epsilon$ enhances the tendency of the $B$ segments to segregate from the solvent. As a result, micelles form at far lower concentrations. We denote this as the "strong segregation regime" in contrast to the "weak segregation regime" of Fig. 1. The lowest volume fraction shown in Fig. 7 ( $\phi$ $=4.2 \times 10^{-5}$, curve a) lies below the CMC. At the twice higher volume fraction $\phi=8.4 \times 10^{-5}$ (curve b) the distribution has become bimodal, indicating the presence of micelles. The large value of $\epsilon$ increases the relaxation time in the Monte Carlo simulations strongly. This increases the statistical noise in the calculations. To obtain the relatively smooth curves in Fig. 7, we applied noise-filtering to the raw histograms. When the volume fraction increases from 8.4 $\times 10^{-5}$ to $2.1 \times 10^{-4}$ (curve $\mathrm{c}$ ) the micellar peak increases but it remains at the same location $\left(N_{\mathrm{agg}} \approx 14\right)$. The growth of the peak indicates that an increasing fraction of the chains occur in aggregates larger than unimers or dimers. A further increase to $\phi=0.021$ (curve d) has no detectable effect on the aggregate size distribution at all. Only for $\phi>0.07$ (curves e and f) do we again see a clear increase in the (average) aggregation number.

These results show that, in the strong segregation regime, the micellar composition remains constant over a wide concentration range (nearly three orders of magnitude in Fig. 7). This is the kind of behavior one expects for "classic" surfactants with a tail that typically consists of $\mathrm{CH}_{2}$ segments. In contrast, in the weak segregation regime the average micelle size increases with the concentration.

Although the simulations were conducted for triblock chains only, one would expect to find similar results for diblock chains. A large number of lattice simulation studies of amphiphilic diblock copolymer chains have been published. However, we have not been able to find a systematic investigation of this issue in the literature dealing with simulations of diblock chains. Nevertheless, one can find results that look similar to those for our triblock chains. For example, Desplat and Care ${ }^{23}$ show results for a system where at a volume fraction of 0.02 (just above the CMC) the average aggregation number is significantly smaller than for $\phi$

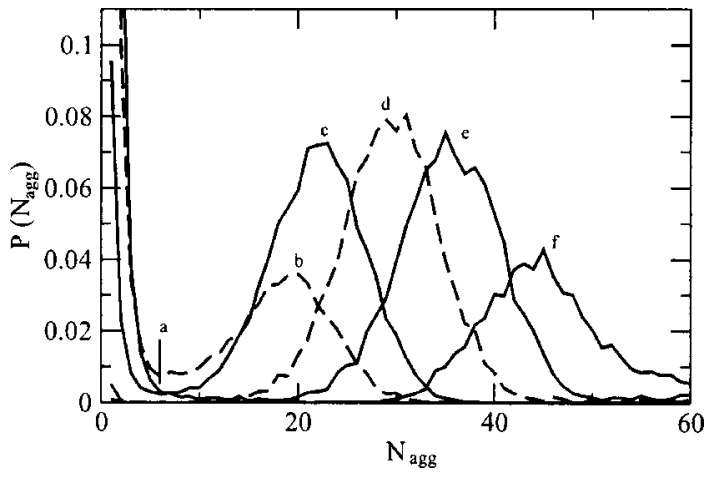

FIG. 8. Aggregate size distribution curves for an $A_{6} B_{6} A_{6}$ triblock chain on a cubic lattice of coordination number 26 . The following interaction parameters were used: $\epsilon_{B S}=\epsilon_{A B}=0.15$ and $\epsilon_{A S}=0$. The amphiphile volume fractions $\phi$ for the curves are: $\phi=0.009$ (curve a); $\phi=0.021$ (b); $\phi=0.072$ (c); $\phi=0.288$ (d); $\phi=0.432$ (e); $\phi=0.576$ (f).

$=0.1$. On the other hand, Mackie et al. present simulations results, where the aggregate size seems to be independent of the amphiphile volume fraction. ${ }^{25}$ However, it is not easy to compare simulations that are based on models with different interaction parameters.

\section{Lattice effects}

Of course, the use of a lattice model may lead to artifacts in the simulation results. In our simulations, we employed a cubic lattice with coordination number 6 . In the literature a large number of simulation studies can be found, using a lattice of either coordination number 6 or coordination number 26. In the latter case segments interact not only with their direct-nearest neighbors but also with their diagonally nearest neighbors. The advantage of this is that the interactions are more isotropic, and a lattice of coordination number 26 may provide a better description of continuous space. Aggregation numbers of micelles tend to be larger in the higher coordination number model. ${ }^{25}$

We conducted a small number of tests using a lattice with coordination number 26 . As the number of contacts in the $z=26$ models is higher than in the $z=6$ model, we should decrease the interaction parameters accordingly: $\epsilon$ $=0.8$ for $z=6$ corresponds to $\epsilon=0.133$ for $z=26$. We find that for $\epsilon=0.133$ the cmc lies significantly higher than the value we find from Fig. 1. For values in the range $\epsilon$ $=0.14-0.15$ we find roughly the same CMC value. In Fig. 8 aggregate size distribution curves are shown for $\epsilon=0.15$. These results are very similar to those obtained in simulations of the low $-z$ model. This suggests that the choice of lattice model has little effect on the qualitative features of our simulations results.

\section{Chemical potential and thermodynamics}

Figure 9 shows the dependence of the chemical potential $\mu$ of the triblock copolymers as a function of volume fraction. The corresponding aggregate-size distribution are shown in Fig. 1. The dots in the graph were obtained using a naive particle-insertion method. The circles correspond to the Rosenbluth scheme. For both cases, on the order of $10^{9}$ test 


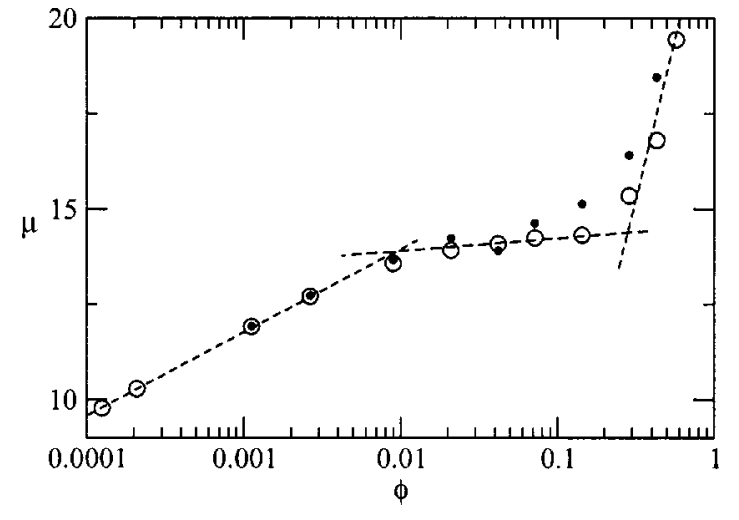

FIG. 9. Chemical potential as a function of amphiphile volume fraction for the system of Fig. 1. The filled small circles were obtained using the direct test chain insertion method. The open circles were computed from the Rosenbluth factor of test chains. The dashed lines are given as guides to the eye only.

chains were generated for each data point. At high volume fractions the uncertainty in the measured value for $\mu$ increases. However, for the Rosenbluth method, the standard deviation remains about as large as the symbols used in the graph. At low volume fractions both methods predict the same value for $\mu$. As is to be expected, the direct insertion method fails at high densities, as the insertion probability becomes very small.

The graph in Fig. 9 is linear at low densities. Then a change in slope occurs around the CMC. Then a second linear regime starts, until we get to a concentration where intermicellar interactions become dominant. In the lowconcentration micellar regime (i.e., the middle regime) the slope of the curve is small, but it clearly remains positive. In the strong segregation regime one expects this slope to become (nearly) zero. We tried to compute $\mu$ for the $\epsilon=1.2$ system, but above the $\mathrm{cmc}$ the statistical errors become too large for this system. The probability that a test chain fits exactly into an existing micelle is small, but such a chain does give a very large contribution to $\mu$. This leads to large fluctuations in the measurements. It would be possible to compute $\mu$ using a more gradual insertion scheme, but we did not attempt that.

For a system at equilibrium, the chemical potential $\mu$ of the monomers is constant throughout the system. If micelles do not interact, we can express $N_{n}$, the number of micelles of size $n$ on a lattice containing $M$ lattice sites as

$$
N_{n}=M \exp \left[-\beta\left(f_{n}-n \mu\right)\right],
$$

where $\beta=1 / k_{B} T$ and $f_{n}$ is the nonideal part of the free energy of the micelle. We can then write

$$
\frac{\beta f_{n}}{n}=\beta \mu-\frac{k_{B} T}{n} \ln \left(\frac{N_{n}}{M}\right) .
$$

Using the data in Figs. 1 and 9, we compute the nonideal free energy per amphiphile $\left(f_{n} / n\right)$, as a function of aggregate size. This is shown in Fig. 10 for three different amphiphile volume fractions. The most striking feature of this figure is that, for large $n, f_{n} / n$ is effectively constant, though weakly dependent on the overall amphiphile concentration. Note that

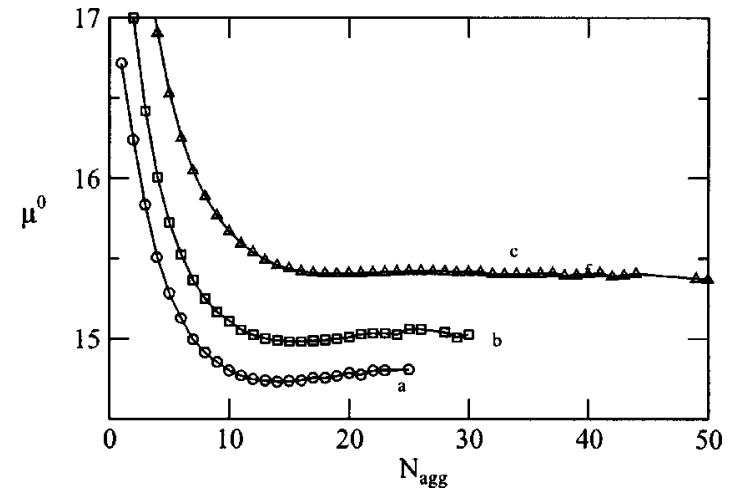

FIG. 10. The intrinsic contribution $\mu_{n}^{\ominus}$ to the amphiphile chemical potential [as defined by Eq. (5)] as a function of the aggregation number. The curves were calculated by combining the data of Figs. 1 and 9 . The three curves refer to the following volume fractions: $\phi=0.072(\mathrm{a}) ; \phi=0.144(\mathrm{~b})$; and $\phi=0.288$ (c).

it then follows from Eq. (4) that, once $\mu$ exceeds $f_{n} / n$, the micelles should grow without bound. This may explain why very large aggregates form at high $\phi$. Moreover, the pronounced dependence of the average micelle size on $\phi$ can be attributed to the rather shallow minimum in $f_{n} / n$.

\section{E. High volume fractions}

Figure 11 shows a two-dimensional cross section through a simulation snapshot at high volume fraction $(\phi$ $=0.576)$. At this volume fraction the aggregate size distribution $P\left(N_{\mathrm{agg}}\right)$ is dominated by clusters that are far larger than those formed just above the CMC (see Fig. 1). Nevertheless, the snapshot shows that relatively small domains are formed with typical length scales on the order of several lattice spacings. For the sake of clarity, only the $B$ segments have been drawn in Fig. 12. The structure formed by these domains can be studied by calculating the radial distribution function $g_{B}(r)$ of the $B$ segments.

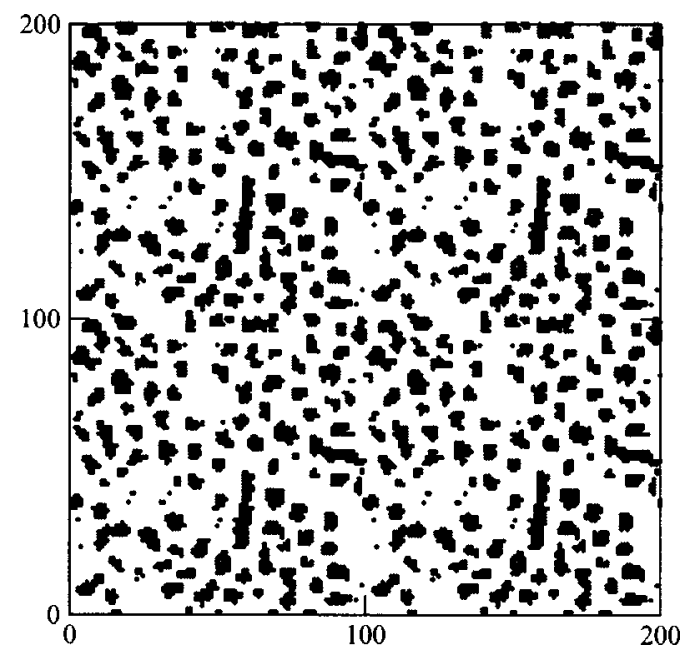

FIG. 11. Two-dimensional cross section through a simulation snapshot of an amphiphile solution at a volume fraction $\phi=0.576$. For the sake of clarity, only the $B$ segments have been drawn. The snapshot is taken from a simulation run using a box size $V=100 \times 100 \times 100$. In order to give a better visual impression of the structure of the system, four periodic images of the cross section are shown. 


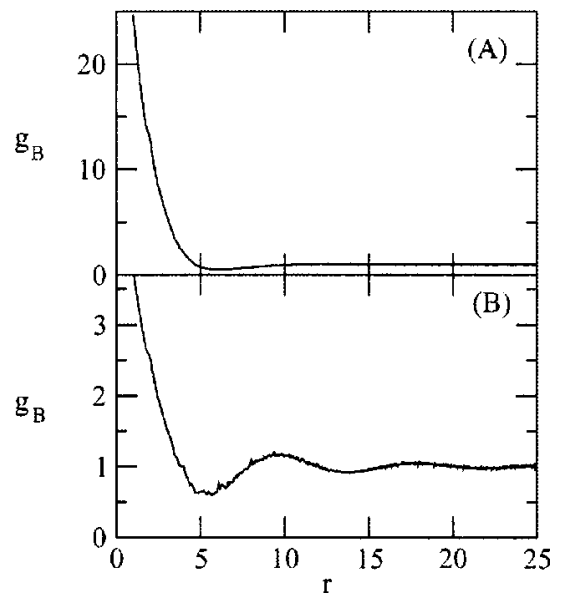

FIG. 12. Radial distribution functions of the $B$ segments $g_{B}(r)$ for $\phi$ $=0.072($ graph A) and $\phi=0.576($ graph B).

Figure 12 shows $g_{B}(r)$ for volume fractions in the range $0.072-0.576$. The curve for $\phi=0.072[$ Fig. 12(A)] is for a system just above the $\mathrm{CMC}$. In this case, the micellar aggregates barely interact. Indeed, the curve in Fig. 12(A) shows that there is little intermicellar ordering at this volume fraction. Strong density correlations exist at short distances $r$ $(r<4.7)$, but this is due to intramicellar correlations. Although the distribution functions in Fig. 12 have been drawn as continuous curves, the function $g_{B}(r)$ is only defined for a finite number of $r$ values which correspond to the distances that exist between any pair of lattice sites on a cubic lattice. As a consequence, some of the apparent features in Fig. 12(A) (e.g., the small shoulder at $r=1.7$ ) are lattice artifacts.

Figure 12(B) shows the radial distribution function for a dense system $(\phi=0.576)$. The behavior of $g(r)$ indicates significant long-range ordering of the $B$ segments. In fact, the correlations have not died down at $r=L / 2$, the maximum length at which $g(r)$ can be measured in a periodic box of diameter $L$. We therefore increased the linear size of the system by a factor $2(L=50)$. In the range where they could be compared, we found no differences between the $g(r)$ for the large and the small system.

The radial distribution function for $\phi=0.576$ has been drawn again. In Fig. 13 we show that $g_{B}(r)$ can be fitted to a function of the form

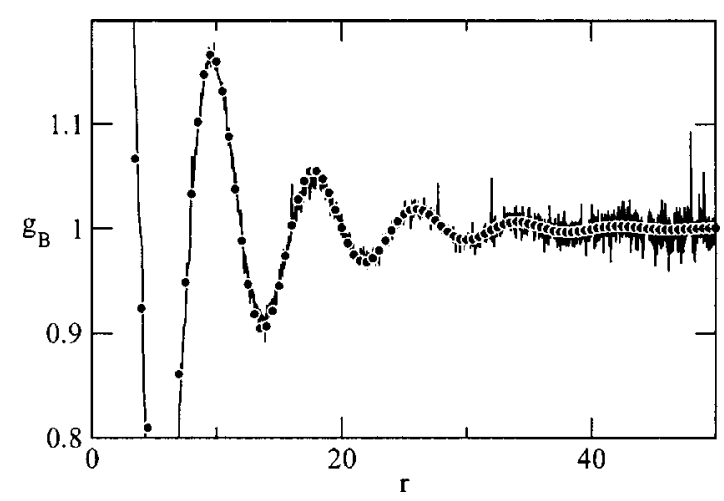

FIG. 13. Radial distribution function for $\phi=0.576$. The curve gives the simulated results. The symbols show the fit of Eq. (6).

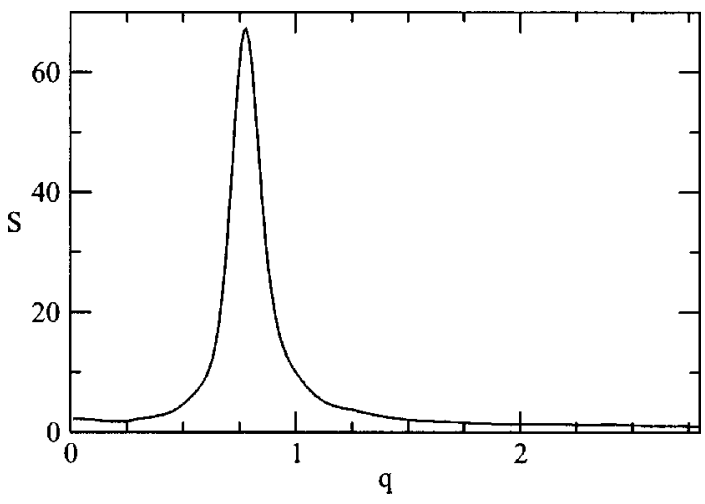

FIG. 14. Structure factor $S(q)$ according to Eq. (7) for $\phi=0.576$.

$$
g(r)=1.0+A \exp (-\lambda r) \cos \left(\frac{2 \pi}{S} r-d\right)
$$

The figure shows an example of such a fit for $\phi=0.576$. This equation gives a good description of the simulated $g(r)$ from the first peak (at $r=9.6$ ) onwards, using the following fitting parameters: $A=0.62, \lambda=0.135, S=8.15$, and $d=1.3$. However, for very small values of $r$ the fit is poor.

In experiments, it is not the radial distribution function of the $B$ segments that is measured directly. Rather, by isotopic labeling and contrasting, it is possible to measure in neutron-scattering experiments the partial structure factor due to the hydrophobic polymer segments. We define a corresponding structure factor $S(q)$ for our system as

$$
S(q)=1+\frac{4 \pi \rho}{q} \int_{0}^{\infty} r \sin (q r)\left[g_{B}(r)-1\right] d r .
$$

This expression was used to obtain the structure factor shown in Fig. 14. For $r<26$ the simulated values for $g_{B}(r)$ were used. For larger $r$ we used an extrapolation based on the analytic fit. In this way, we minimize artifacts due to finitesize effects. The dominant feature of $S(q)$ is the peak at $q$ $=0.78$. This peaks compares well with a spacing of 8.15 (lattice units) between the oscillations in the radial distribution function.

\section{F. Composite hard-sphere model}

Let us next consider to what extent the micellar system can be described by a hard-sphere model. We consider hard spheres with a diameter $d$ equal to unity. To represent the distinction between $A$ and $B$ segments, we divide the sphere into an inner core with a diameter $d_{\text {core }}(<d)$ and an outer corona. The core is supposed to consist of $B$, the corona of $A$. Note that this distinction between $A$ and $B$ parts does not affect the interactions between the particles.

We performed Monte Carlo simulations of this hardsphere model and computed the radial distribution function $g_{\text {core }}(r)$ of the $B$ particles. We denote the hard-sphere number density by $\rho \equiv N / V$, and the volume fraction by $\phi \equiv \pi / 6$ $\times \rho$.

Figure 15 shows the results of a simulation at a number density $\rho=0.95$ and a core diameter $d_{\text {core }}=0.75$ (using a volume $V=10^{3}$ ). We fit our simulation data to an expression 


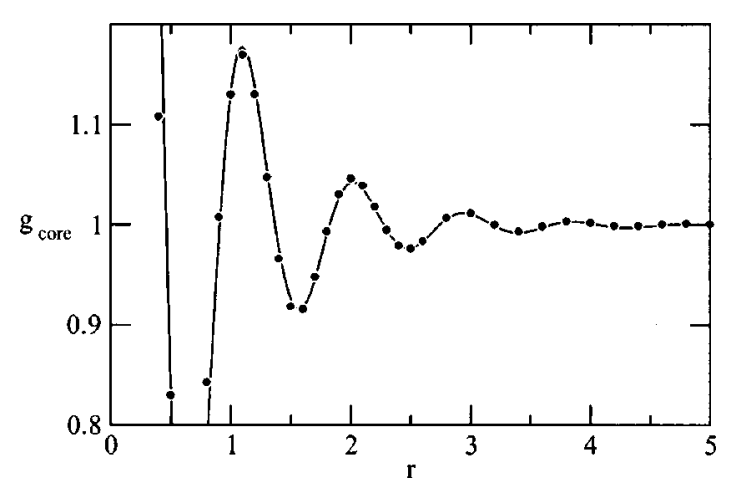

FIG. 15. The core radial distribution function $g_{\text {core }}(r)$ for the composite hard-sphere model with $\rho=0.95$ and $d_{\text {core }}=0.75$. The full curve shows the simulated data and the symbols are calculated using the analytic fit of Eq. (6).

of the form given by Eq. (6), yielding $A=0.80, \lambda=0.14, S$ $=0.92$, and $d=1.4$. Beyond the first maximum at $r \approx 1$, Eq. (6) provides a good fit to the simulated curve.

The values for $\rho$ and $d_{\text {core }}$ used in the simulation of Fig. 15 were chosen after several trial runs to find the parameters that gave the best agreement with the lattice model simulations. Figure 16 shows a direct comparison between both models. The radial distribution curves $g_{B}$ and $g_{\text {core }}$ are drawn as a function of the scaled distance $r / S$. In both cases $S$ is the period of the oscillation of $g(r)$ [Eq. (6)]. The agreement between both models in Fig. 16 is surprisingly good, even at distances smaller than the hard-sphere diameter. Such an agreement between the micellar and hard-sphere models is by no means intuitively obvious, in particular as Figs. 1 and 11 show that the micelles are really quite different from monodisperse hard spheres. In fact, to address this last point, we performed simulations on a polydisperse hard-sphere model with a log-normal ${ }^{47,48}$ distribution that corresponded to the distribution of micelle sizes. Surprisingly, such a polydisperse hard-sphere model gave worse agreement with the lattice simulations than the monodisperse model.

Experimentally, bcc and fcc ordering has been observed for binary mixtures of several kinds of pluronics and water. The question arises if such a crystallization can be found in the lattice model. It seems likely that this is not the case for

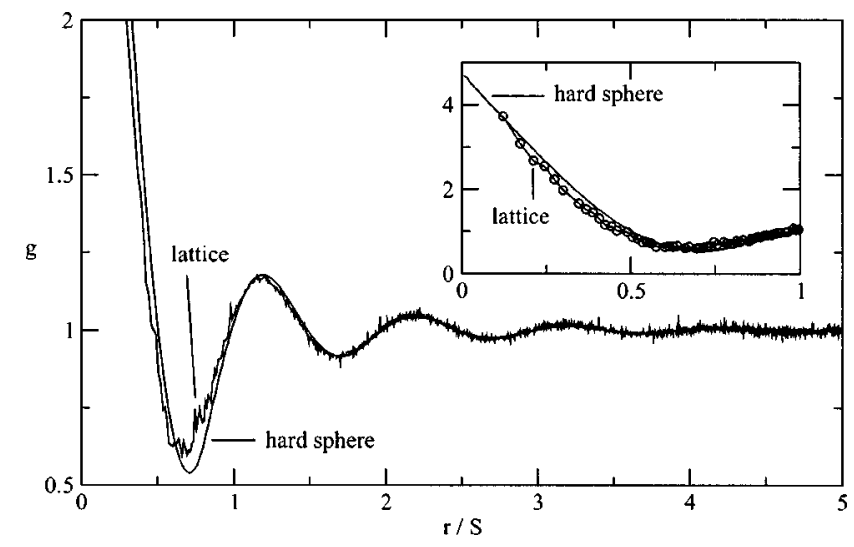

FIG. 16. Comparison of the (scaled) radial distribution functions from the lattice model and the hard-sphere model.

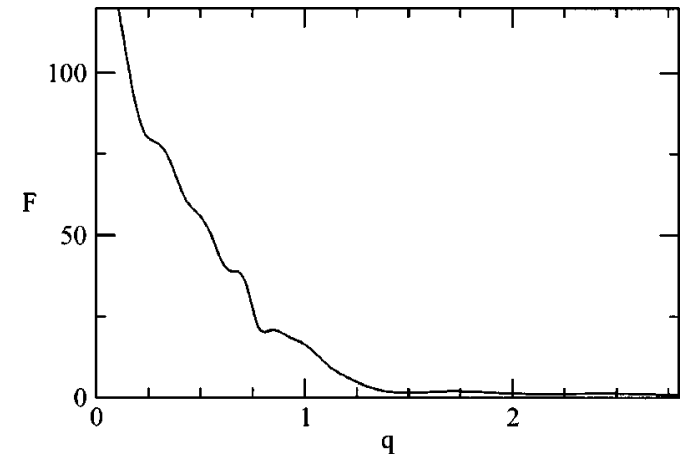

FIG. 17. Form factor $F(q)$ (as defined in the text) for the $\phi=0.576$ system.

the concentrations that we studied. In fact, the highest density that we considered $(\phi=0.576)$ maps onto a hard sphere fluid with a density $\rho=0.95\left(\phi_{\mathrm{HS}}=0.497\right)$. This density is marginally higher than the freezing density. ${ }^{49}$ So, strictly speaking, our hard-sphere reference fluid is metastable with respect to the solid. However, hard-sphere reference systems at slightly lower densities described the micellar system almost as well. Hence we can only state that the hard-sphere system that models our densest micellar solution, is very close to freezing.

Mortensen $^{6}$ analyzed small-angle neutron-scattering data of PEO-PPO-PEO triblock copolymers by extracting the micellar form factor from the scattering function at low concentrations. Subsequently, the scattering at high concentration was modeled as the product of this form factor and the structure factor of a hard-sphere dispersion. This analysis assumes that the size and shape of the micelles do not change with copolymer concentration. In our simulations, this assumption clearly does not hold. This becomes apparent by comparing the radial distribution functions at small $r$ (determined by the intra-aggregate structure) for low and high volume fractions [Figs. 12(A) and (B)]. As an additional test, we used the hard-sphere model that fitted the high-density lattice model to simulate the micellar solution at $\phi=0.072$. To this end, we changed the hard-sphere density to $\rho=0.072 / 0.576$ $\times 0.95=0.12$. This procedure yielded very poor agreement with the lattice simulations for $\phi=0.072$. By treating $\rho$, $d_{\text {core }}$, and the scaling parameter $S$ as fitting parameters in the hard-sphere model one can get a slightly better fit of the lattice model results. Nevertheless, for low amphiphile concentrations it is not possible to achieve a reasonable agreement between the radial distribution functions $g_{B}(r / S)$ (lattice model) and $g_{\text {core }}(r / S)$ (hard-sphere model).

Still, at high densities, the (monodisperse) hard-sphere model appears to work well. We therefore assumed that the structure factor of the $B$ segments in the lattice model can be expressed as the product of a hard-sphere structure factor and a micellar form factor. To this end, we divided the $(B)$ segmental structure factor by the structure factor of hard spheres (we used Baxter's analytical solution of the hard-sphere Percus-Yevick equation ${ }^{50}$ ). Figure 17 shows the resulting ratio, which can be considered as the form factor of the effective composite hard spheres that represent our micellar model system. 


\section{CONCLUSIONS}

The present study shows that a simple (lattice) model of a triblock copolymer can, at least qualitatively, account for the experimentally observed density dependence of the structural properties of such systems. In particular, we find that for "weakly segregating" triblock copolymers, the size of the micelles depends noticeably on the amphiphile concentration.

At high volume fractions we find that the structure of the amphiphilic system can be mapped onto that of a high density, monodisperse hard-sphere fluid. This is rather surprising, as the micellar system itself is quite polydisperse. Even though a hard-sphere model allows us to describe the dense micellar system, the same model fails at low densities. Hence it is incorrect to assume that the scattering intensity of such micellar systems can be represented by the product of a density-dependent hard-sphere structure factor and a densityindependent form factor.

\section{ACKNOWLEDGMENTS}

The work of the FOM Institute is part of the research program of FOM, and is made possible by financial support from NWO.

${ }^{1}$ P. Alexandridis and T. A. Hatton, Colloids Surf., A 96, 1 (1995).

${ }^{2}$ P. Alexandridis, Curr. Opin. Colloid Interface Sci. 2, 478 (1997).

${ }^{3}$ K. Mortensen, Colloids Surf., A 183, 277 (2001).

${ }^{4}$ D. R. Nelson and J. Toner, Phys. Rev. B 24, 363 (1981).

${ }^{5}$ P. J. Steinhardt, D. R. Nelson, and M. Ronchetti, Phys. Rev. B 28, 784 (1983).

${ }^{6}$ K. Mortensen, J. Phys.: Condens. Matter 8, A103 (1996).

${ }^{7}$ K. Mortensen, W. Brown, and B. Nordén, Phys. Rev. Lett. 13, 2340 (1992).

${ }^{8}$ P. N. Hurter, J. M. H. M. Scheutjens, and T. A. Hatton, Macromolecules 26, 5030 (1993); 26, 5592 (1993).

${ }^{9}$ P. Linse, Macromolecules 26, 4437 (1993); 27, 2685 (1994); 27, 6404 (1994).

${ }^{10}$ P. Linse, J. Phys. Chem. 97, 13896 (1993).

${ }^{11}$ J. Noolandi, A.-C. Shi, and P. Linse, Macromolecules 29, 5907 (1996).

${ }^{12}$ M. Svensson, P. Alexandridis, and P. Linse, Macromolecules 32, 637 (1999).

${ }^{13}$ J. C. Shelley and M. Y. Shelley, Curr. Opin. Colloid Interface Sci. 5, 101 (2000).

${ }^{14}$ K. Binder and M. Müler, Curr. Opin. Colloid Interface Sci. 5, 315 (2000).

${ }^{15}$ R. G. Larson, L. E. Scriven, and H. T. Davis, J. Chem. Phys. 83, 2411 (1985).
${ }^{16}$ C. M. Care, J. Chem. Soc., Faraday Trans. 1 83, 2905 (1987).

${ }^{17}$ R. G. Larson, J. Chem. Phys. 89, 1642 (1988); 91, 2479 (1989); 96, 7904 (1992).

${ }^{18}$ K. Rodrigues and W. L. Mattice, J. Chem. Phys. 94, 761 (1991); 95, 5341 (1991).

${ }^{19}$ D. Brindle and C. M. Care, J. Chem. Soc., Faraday Trans. 88, 2163 (1992).

${ }^{20}$ Y. M. Wang, W. L. Mattice, and D. H. Napper, Langmuir 9, 66 (1993).

${ }^{21}$ Y. J. Zhan and W. L. Mattice, Macromolecules 27, 677 (1994).

${ }^{22}$ C. M. Wijmans and P. Linse, J. Phys. Chem. 100, 12583 (1996).

${ }^{23}$ J.-C. Desplat and C. M. Care, Mol. Phys. 87, 441 (1996).

${ }^{24}$ P. H. Nelson, G. C. Rutledge, and T. A. Hatton, J. Chem. Phys. 107, 10777 (1997).

${ }^{25}$ A. D. Mackie, A. Z. Panagiotopoulos, and I. Szleifer, Langmuir 13, 5022 (1997).

${ }^{26}$ L. A. Rodriguez-Guadarrama, S. K. Talsania, K. K. Mohanty, and R. Rajagopalan, Langmuir 15, 437 (1999).

${ }^{27}$ C. M. Care and T. Dalby, Europhys. Lett. 45, 38 (1999).

${ }^{28}$ M. A. Floriano, E. Caponetti, and A. Z. Panagiotopoulos, Langmuir 15, 3143 (1999).

${ }^{29}$ M. Girardi and W. Figueiredo, J. Chem. Phys. 112, 4833 (2000).

${ }^{30}$ M. P. Pépin and M. D. Whitmore, Macromolecules 33, 8644 (2000); 33, 8654 (2000).

${ }^{31}$ A. Bhattacharya and S. D. Mahanti, J. Phys.: Condens. Matter 12, 6141 (2000).

${ }^{32}$ W. Kenward and M. D. Whitmore, J. Chem. Phys. 116, 3455 (2002).

${ }^{33}$ K. Rodrigues and W. L. Mattice, Langmuir 8, 456 (1992).

${ }^{34}$ L. Xing and W. L. Mattice, Macromolecules 30, 1711 (1997); Langmuir 14, 4074 (1998)

${ }^{35}$ S. H. Kim and W. H. Jo, Macromolecules 34, 7210 (2001); J. Chem. Phys. 117, 8565 (2002).

${ }^{36}$ A. Z. Panagiotopoulos, M. A. Floriano, and S. K. Kumar, Langmuir 18, 2940 (2002).

${ }^{37}$ A. Milchev and K. Binder, Langmuir 15, 3232 (1999).

${ }^{38}$ P. Alexandridis and A. Hatton, Colloids Surf., A 96, 1 (1995).

${ }^{39}$ P. H. Verdier and W. H. Stockmayer, J. Chem. Phys. 36, 227 (1962).

${ }^{40}$ N. Metropolis, A. W. Rosenbluth, M. N. Rosenbluth, A. N. Teller, and E. Teller, J. Chem. Phys. 21, 1087 (1953).

${ }^{41}$ B. Widom, J. Chem. Phys. 39, 2802 (1963).

${ }^{42}$ D. Frenkel and B. Smit, Understanding Molecular Simulations: From Algorithms to Applications, 2nd ed. (Academic, San Diego, 2001).

${ }^{43}$ J. Harris and S. A. Rice, J. Chem. Phys. 88, 1298 (1988).

${ }^{44}$ J. I. Siepmann, Mol. Phys. 70, 1145 (1990).

${ }^{45}$ M. N. Rosenbluth and A. W. Rosenbluth, J. Chem. Phys. 23, 356 (1955).

${ }^{46}$ E. Ruckenstein and R. Nagarajan, J. Chem. Phys. 79, 2622 (1975).

${ }^{47}$ C. Tanford, J. Phys. Chem. 78, 2469 (1974).

${ }^{48}$ D. Frenkel, R. J. Vos, C. G. de Kruif, and A. Vrij, J. Chem. Phys. 84, 4625 (1986).

${ }^{49}$ W. G. Hoover and F. H. Ree, J. Chem. Phys. 49, 3609 (1968).

${ }^{50}$ R. J. Baxter, Aust. J. Phys. 21, 563 (1968). 